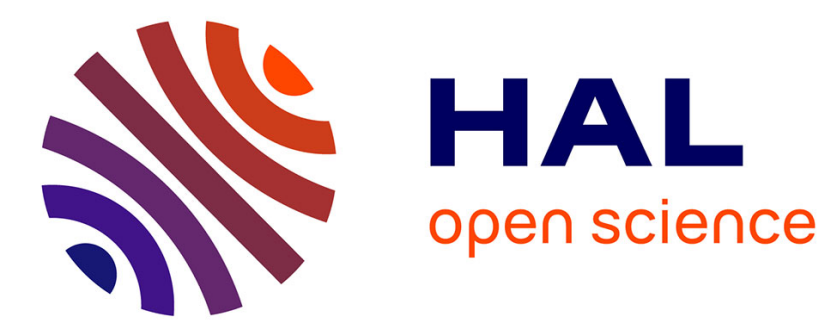

\title{
Time-reversal focusing of therapeutic ultrasound on targeted microbubbles
}

Olivier Couture, Jean-François Aubry, Mickaël Tanter, Mathias Fink

\section{To cite this version:}

Olivier Couture, Jean-François Aubry, Mickaël Tanter, Mathias Fink. Time-reversal focusing of therapeutic ultrasound on targeted microbubbles. Applied Physics Letters, 2009, 94 (17), pp.173901. 10.1063/1.3126039 . hal-02481794

\section{HAL Id: hal-02481794 \\ https://hal.science/hal-02481794}

Submitted on 17 Feb 2020

HAL is a multi-disciplinary open access archive for the deposit and dissemination of scientific research documents, whether they are published or not. The documents may come from teaching and research institutions in France or abroad, or from public or private research centers.
L'archive ouverte pluridisciplinaire HAL, est destinée au dépôt et à la diffusion de documents scientifiques de niveau recherche, publiés ou non, émanant des établissements d'enseignement et de recherche français ou étrangers, des laboratoires publics ou privés. 


\section{Time-reversal focusing of therapeutic ultrasound on targeted microbubbles}

Cite as: Appl. Phys. Lett. 94, 173901 (2009); https://doi.org/10.1063/1.3126039

Submitted: 29 October 2008 . Accepted: 06 April 2009 . Published Online: 27 April 2009

Olivier Couture, Jean-François Aubry, Mickael Tanter, and Mathias Fink

\section{ARTICLES YOU MAY BE INTERESTED IN}

Sono-activated ultrasound localization microscopy

Applied Physics Letters 103, 174107 (2013); https://doi.org/10.1063/1.4826597

"Ultrasonic stars" for time-reversal focusing using induced cavitation bubbles Applied Physics Letters 88, 034102 (2006); https://doi.org/10.1063/1.2162700

Acoustic imaging with time reversal methods: From medicine to NDT

AIP Conference Proceedings 1650, 13 (2015); https://doi.org/10.1063/1.4914591

Lock-in Amplifiers Find out more today

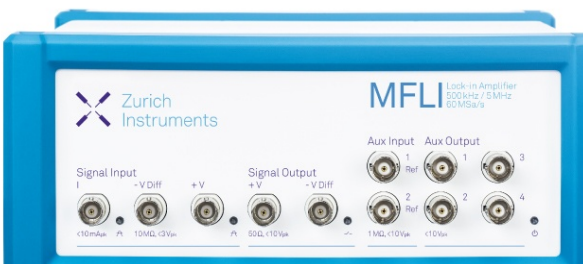

Zurich

- Instruments 


\title{
Time-reversal focusing of therapeutic ultrasound on targeted microbubbles
}

\author{
Olivier Couture, ${ }^{1,2, a)}$ Jean-François Aubry, ${ }^{1}$ Mickael Tanter, ${ }^{1,3}$ and Mathias Fink ${ }^{1}$ \\ ${ }^{1}$ Institut Langevin Ondes et Images (CNRS UMR 7587), ESPCI, Paris 75005, France \\ ${ }^{2}$ Fondation Pierre-Gilles de Gennes, France \\ ${ }^{3}$ INSERM, France
}

(Received 29 October 2008; accepted 6 April 2009; published online 27 April 2009)

\begin{abstract}
Targeted microbubbles bind specifically to molecular markers of diseases and their unique acoustic signature is used to image cellular processes in vivo. The ability of time-reversal processing to focus waves through heterogeneities on such targeted microbubbles is demonstrated. For this purpose, microbubbles were deposited on a gelatin phantom and their specific signal was recorded by a high intensity ultrasonic array. The amplified time-reversed signal was re-emitted and shown to focus back in the region where the bound microbubbles were present. This proof of concept emphasizes that molecular-time-reversal focusing could guide energy deposition on early, diffuse, or metastatic disease. (C) 2009 American Institute of Physics. [DOI: 10.1063/1.3126039]
\end{abstract}

Ultrasound therapy can treat various pathologies such as solid tumors, arteriosclerosis, and hemorrhage. ${ }^{1}$ Absorption of ultrasound energy not only leads to tissue heating but also to cavitation and sonoporation. ${ }^{2}$ These effects can necrose tissue or induce apoptosis, ${ }^{3}$ facilitate the passage of drugs ${ }^{4}$ or erode thrombi in vivo. ${ }^{5}$ In homogeneous media, the energy deposition is optimal at the focus of the ultrasonic transducer, determined either by the geometry of the source or the electronic delays applied on the elements of an array. The focal zone can be placed deep into tissue and moved to treat a diseased region highlighted by prior ultrasound imaging, magnetic resonance imaging, or computed tomography. However, these imaging methods are, currently, mainly sensitive toward changes in physical properties of tissues. Treatment based on the molecular and cellular processes underlying cancer and arteriosclerosis are likely to be more specific to the early onset of these diseases.

Such molecular specificity has been attained in ultrasound imaging by exploiting targeted contrast agents. ${ }^{6}$ These agents, usually bubbles a few microns in diameter, are retained in a tumor or thrombus through the antibodies or ligands present on their surface. These microbubbles have a specific acoustic response allowing ultrasound imaging to distinguish them from tissue or blood. For instance, microbubbles emits detectable harmonics when insonified by a monochromatic incident wavefield. ${ }^{7}$ They can also be disrupted, which makes differentiation techniques very sensitive to microbubbles. ${ }^{8}$ In fact, the ultrasound signature of a single bound microbubble can be detected. ${ }^{9}$ Targeting of these contrast agents causes these echoes to originate from a region of disease-specific molecular expression.

The Laboratoire Ondes et Acoustique previously exploited time-reversal acoustics ${ }^{10,11}$ to focus waves on cavitation bubbles induced within tissue. ${ }^{12}$ This method was shown to efficiently correct aberrations and improve focusing of ultrasound therapy. A similar technique can be applied to targeted contrast agents. Using the ability of time-reversal to refocus an amplified ultrasound wave toward its initial source, we present a method to restrict energy deposition on localized or extended areas of specific biomarkers expres-

${ }^{a)}$ Electronic mail: olicou@gmail.com. sion. Beyond the important issue of specificity, targeted contrast agents could also provide an efficient way to correct for beam aberrations induced by heterogeneities encountered by the wave on its travel path.

An in vitro targeting phantom was designed to demonstrate specific focusing of ultrasound on bound microbubbles (Fig. 1). Gelatin (5\%) and biotin (1\%) were mixed into water and poured in plastic Petri dishes. Microbubbles covered with avidin (Bracco Research SA) were diluted (1/10), mixed with black ink and deposited as $15 \mu \mathrm{l}$ droplet on the gel surface. ${ }^{13}$ The microbubbles interacted with the surface of the gel through the avidin-biotin complex and, after washing, left a disk $5 \mathrm{~mm}$ in diameter populated with bound microbubbles. Surface density of microbubbles was estimated to attain 70 microbubbles $/ \mathrm{mm}^{2}{ }^{13}$ The plates were then installed in a water tank such that the gelatin surface was coplanar with the focal plane of a 70-elements high-intensity

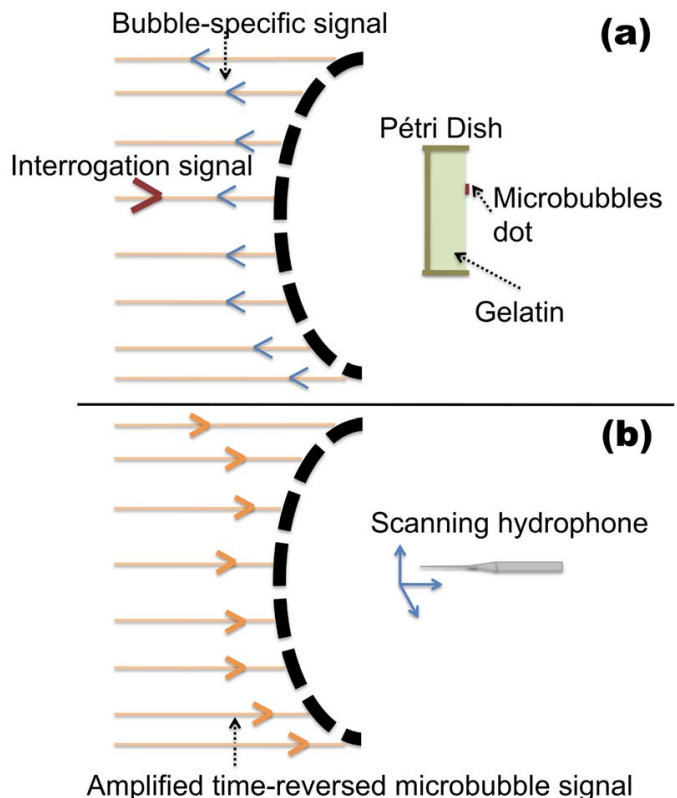

FIG. 1. (Color online) Time-reversal on a dot of microbubbles. (a) The echo of an interrogation pulse is collected before and after the disruption of the microbubbles. (b) The time-reversed signal of the microbubbles is amplified, emitted, and measured by a hydrophone. 
(a)

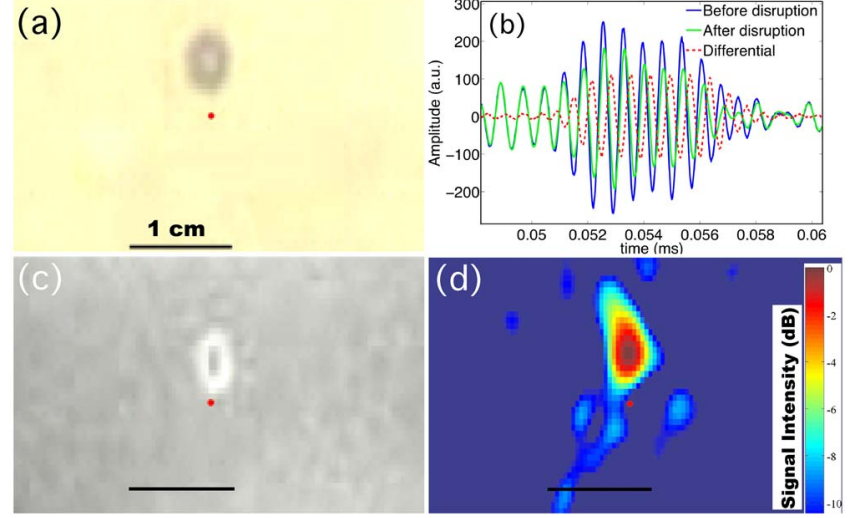

FIG. 2. (Color online) (a) Photography of the dyed microbubbles dot. (b) Echo of a weak interrogation pulse on the gelatin surface on a single element. (c) Photography of the color change on the thermosensitive paper. (d) Acoustic field collected by the hydrophone. The red dot is the geometric focal point, the black line is $10 \mathrm{~mm}$ long and the colorbar scale is $\mathrm{dB}$ relative to the maximum.

focused ultrasound array. Each element of the array were emitter-receiver tuned at $1 \mathrm{MHz}$ and driven by a fully programable electronic board relying on a $30 \mathrm{MHz}$ sampling frequency analog transmitter. A micrometric step motor moved the microbubbles dots with respect to the geometric focus of the transducer to determine steering capabilities.

The specific signal of the microbubbles was collected through their disruption. At first, a low pressure imaging pulse was emitted by a single transducer to avoid a priori focusing. The corresponding echoes from the plate and the microbubbles were collected by the entire array. Then, a high amplitude pulse was emitted by a high intensity focused ultrasound array, with sufficient pressure to disrupt the microbubbles (1.5 MPa). Finally, a new series of imaging pulses were collected. The difference between the signals before and after disruption was assumed to be specific to microbubbles [Fig. 2(b)]. These differential signals were recorded and saved. The experiment was reproduced for several positions of the microbubbles dot within the acoustic field and for several patterns of dots. This differential sequence of ultrasonic insonifications was performed in a few milliseconds and would thus be insensitive to motion artifacts in a clinical configuration.

The position of the dots of microbubbles was recorded with a camera aligned along the transducer axis. Figure 2(a) shows a picture of the disk of dyed microbubbles with respect to the focal point of the transducer. Such pictures were thresholded and the patterns were used as an overlay on the acoustic field measurement displayed in Figs. 3 and 4.

A coarse two-dimensional (2D) mapping of the acoustic energy refocusing was made using a thermosensitive paper, which replaced the gelatin dish in the water tank. The recorded echoes of the microbubbles, for each position, were time reversed, elongated, and amplified to create an emission pattern. These patterns were then emitted by the ultrasound array. Direct heating due to focused ultrasound waves could be detected through color changes of the thermosensitive paper. It was observed that the region of increased temperature was concomitant to the corresponding position of the microbubbles [Fig. 2(c)].

More precise acoustic measurements were performed by scanning a hydrophone (Onda, HNZ 0200) in the focal plane

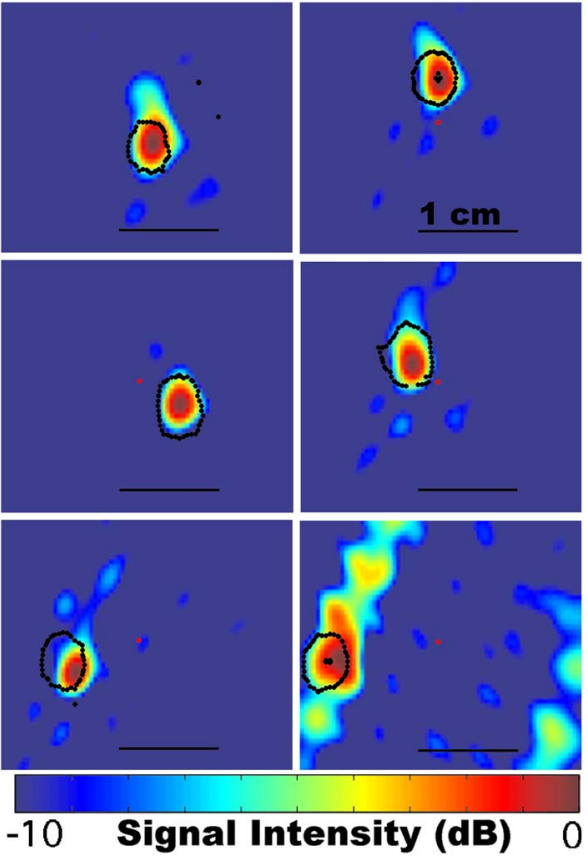

FIG. 3. (Color online) Acoustic field collected by the hydrophone for dots of microbubbles at different positions. The colorbar scale is $\mathrm{dB}$ relative to the maximum. The black border corresponds to the position of the microbubbles dot as observed optically.

of the transducer ( $0.4 \mathrm{~mm}$ steps in $x, y$ plane). The emission patterns, only a few cycles in length, were sent and the resulting acoustic field was measured at each point. As shown in Fig. 2(d), the acoustic field is maximum in the area where the microbubbles were bound on gelatin surface. It demonstrated that ultrasound therapy can be guided by the echoes of the microbubbles.

When the microbubbles dot was moved around the geometric focus of the array, the therapeutic beam was simply redirected toward the new position (Fig. 3). In our specific experiment, the array was able to steer the beam within 1.5 $\mathrm{cm}$ from the geometric focus. However, this limitation, along with the observed sidelobes, depends solely on the physical characteristics of the array such as the number and geometry of the transducers, the emission frequency, the aperture and focal distance.
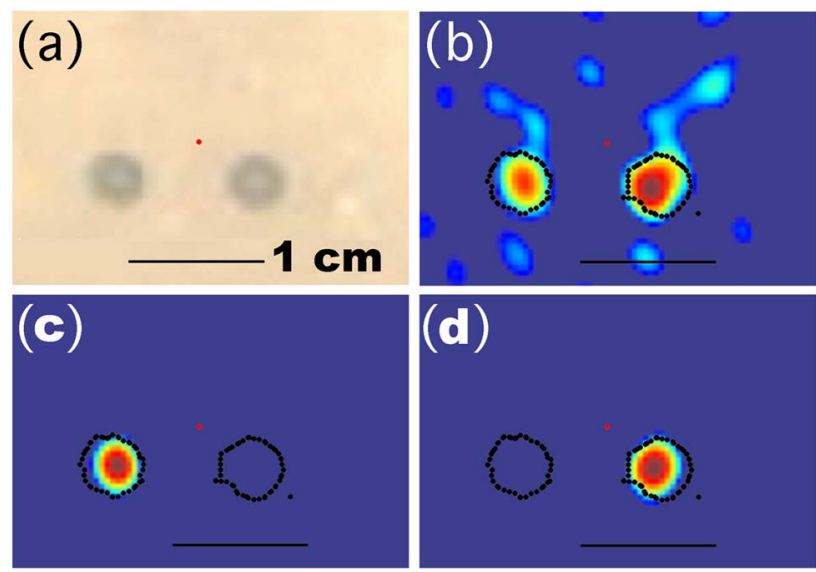

FIG. 4. (Color online) (a) Photography of the two dyed microbubbles dot (b) Acoustic field collected by the hydrophone. [(c) and (d)] Separation of the two microbubbles dots. Acoustic field after geometric projection and selection. 
During molecularly targeted therapy, it is likely that the targeted microbubbles will accumulate over a region larger than the wavelength or in multiple metastasis. Figures 4(a) and 4(b) show how time-reversal manages to refocus simultaneously on two separate dots of microbubbles. It emphasizes the ability of time-reversal processing to build a focal pattern whose shape instantaneously fits the targeted microbubbles area. Mild temperature elevation or cavitationdependent effect could thus be achieved in multiple distinct areas. However, as the treatment volume increases, part of the antennae gain is lost and treatment time has to be increased in order to accumulate sufficient thermal doses for treatment.

To allow separate treatment of each dot, the treated zones were spatially divided using beamforming. The emission pattern was convoluted with the transfer matrix acquired in a homogeneous medium. The signals coming from the each side of the field were separated on the resulting image using a convolution by a $2 \mathrm{D}$ mask and converted back to emission patterns by convoluting with the transpose of the transfer matrix. As shown in Figs. 4(c) and 4(d), these two emission patterns focused successively on each dot and high acoustic pressures were retrieved. More intricate pattern of microbubbles could also be treated by selecting individual part of the echoes and treating point-by-point.

This study demonstrated that the echoes from bound microbubbles can be exploited for direct time-reversal ultrasound therapy. Thus, ultrasonic heating or cavitation can be targeted to diffuse or early disease based on its molecular expression and not only on the modification of its physical properties. This could benefit the treatment of metastasis and infiltrating tumors. Moreover, contrarily to drug-carrying mi- crobubbles, "molecular-focusing" decouples therapeutic dose from the contrast agents. After the bubble-specific signals are recorded, the technique does not require the microbubbles to be present and the deposited energy is purely controlled by ultrasound. Because this technique does not require prior imaging, it can easily be made iterative and treatment can be repeated as long as microbubbles binds in large amounts in a region. However, molecular-focusing is limited to highly specific microbubbles targeting which can only improve as our knowledge of the cellular pathways of disease progress.

The authors thank Bracco Research SA for kindly providing the targeted microbubbles.

${ }^{1}$ G. ter Haar, Prog. Biophys. Mol. Biol. 93, 111 (2007).

${ }^{2}$ C. X. Deng, F. Sieling, H. Pan, and J. Cui, Ultrasound Med. Biol. 30, 519 (2004).

${ }^{3}$ N. Vykhodtseva, N. McDannold, and K. Hynynen, Ultrasound Med. Biol. 32, 1923 (2006).

${ }^{4}$ J. J. Choi, M. Pernot, S. A. Small, and E. Konofagou, Ultrasound Med. Biol. 33, 95 (2007).

${ }^{5}$ R. J. Siegel and H. Luo, Ultrasonics 48, 312 (2008).

${ }^{6}$ P. A. Dayton and J. J. Rychak, Front. Biosci. 12, 5124 (2007).

${ }^{7}$ P. N. Burns and S. R. Wilson, Ultrasound Q. 22, 5 (2006).

${ }^{8}$ K. Wei, D. M. Skyba, C. Firschke, A. R. Jayaweera, J. R. Lindner, and S. Kaul, J. Am. Coll. Cardiol. 29, 1081 (1997).

${ }^{9}$ A. L. Klibanov, P. T. Rasche, M. S. Hughes, J. K. Wojdyla, K. P. Galen, and J. H. Wible G.H. Brandenburger, Invest. Radiol. 39, 187 (2004).

${ }^{10}$ M. Fink, D. Cassereau, A. Derode, C. Prada, P. Roux, M. Tanter, J.-L. Thomas, and F. Wu, Rep. Prog. Phys. 63, 1933 (2000).

${ }^{11}$ M. Fink, G. Montaldo, and M. Tanter, Annu. Rev. Biomed. Eng. 5, 465 (2003).

${ }^{12}$ M. Pernot, G. Montaldo, M. Tanter and M.Fink, Appl. Phys. Lett. 88, 034102 (2006).

${ }^{13}$ O. Couture, M. Sprague, E. Cherin, P. N. Burns, and F. S. Foster, IEEE Trans. Ultrason. Ferroelectr. Freq. Control 56, 536 (2009). 